Methods An abdominal paracentesis teaching programme was introduced from October 2016 to July 2017. An online 'Survey Monkey' questionnaire assessed CMTs pre-existing experience in therapeutic procedures including ascitic drain insertion, their confidence performing procedures and availability of opportunities to practice with appropriate supervision. An online 'Doodle' poll offered CMTs weekly 'drop in' slots to attend the RFH Planned Investigation and Treatment Unit where patients attended for elective paracentesis. CMTs were given one-to-one teaching on ascitic drain insertion technique, including the use of bedside ultrasound to identify appropriate insertion sites. The CMTs were then supervised inserting ascitic drains on consenting, elective patients by an experienced clinician and signed off for Direct Observation of Procedural Skills (DOPS) assessments. A Whatsapp group allowed informal feedback and slot swaps amongst the CMTs. An online survey at the end of the programme sought feedback on how valuable the trainees had found it in improving procedural confidence and obtaining required DOPS. No complications were reported by supervisors during supervised drain insertion.

\begin{tabular}{|c|c|c|}
\hline & $\begin{array}{l}\text { Pre-programme } \\
(\mathrm{n}=22)\end{array}$ & $\begin{array}{l}\text { Post-programme } \\
(\mathrm{n}=14)\end{array}$ \\
\hline Respondents (out of 22 CMT's) & $19 / 22$ & $14 / 14$ \\
\hline Requisite experience (performed $>2$ times) & $32 \%$ & $100 \%$ \\
\hline Difficult/unable to gain experience previously & $53 \%$ & - \\
\hline $\begin{array}{l}\text { Confidence performing paracentesis } \\
\text { independently }\end{array}$ & $26 \%$ & $64 \%$ \\
\hline $\begin{array}{l}\text { E-portfolio signed off by experienced } \\
\text { clinician }\end{array}$ & $79 \%$ & $100 \%$ \\
\hline
\end{tabular}

Conclusion The introduction of a bespoke CMT paracentesis training programme at $\mathrm{RFH}$ helped trainees safely gain experience and confidence in ascitic drain insertion. The weekly opportunities throughout the year ensured all CMTs received the requisite number of DOPS assessments from experienced supervisors. Confidence performing the procedure increased significantly. However, following the programme, 36\% of CMTs still lacked confidence performing paracentesis independently. Future programs should explore additional opportunities for simulation and real patient practice to further improve this. This model can be replicated in any Trusts with elective ascitic drain services, improving ARCP outcomes and increasing procedural confidence for CMTs progressing to medical registrar roles nationally.

\section{PTH-137 ENDOSCOPY EXPERIENCE FOR UK TRAINEES IN KHARTOUM, SUDAN: SOUTH YORKSHIRE EXPERIENCE}

Thazin Min*, Ammar Al-Rifaie, Karen Smith, Paul Hurlstone, Abdelmounem Abdo, Elmuhtady Said, Mark Donnelly. South Yorkshire-Sudan Endoscopy Training group

\subsection{6/gutjnl-2018-BSGAbstracts.536}

Introduction There is increasing interaction between UK gastroenterologists and those in resource-challenged countries. Our group has established links over 7 years with the Sudanese Society of Gastroenterology (SSG) and have previously provided training and support for consultant trainers, trainee endoscopists and endoscopy nurses, as well as supporting the annual SSG conference. Our host hospital, Ibn Sina (IS) is the national gastrointestinal centre and a World Gastroenterology Organisation training centre. Up to 60 cases of oesophageal varices are admitted per week. This is accounted for by the endemic nature of schistosomiasis and a high prevalence of viral hepatitis. This year, we arranged for 2 UK StRs to travel to Sudan to experience therapy for bleeding varices and to work in a resource-limited environment.

Methods Two ST4 registrars were selected from the South Yorkshire region after a competitive application process. Funding was provided by UK based Sudanese doctors, SSG and local research funds. They performed UGI endoscopy from 8th - 11th of January 2018 at IS. The trainees were JAG accredited for diagnostic gastroscopy (OGD) only, therefore all therapeutic interventions were supervised. Prior experience of one registrar was 370 OGDs with 4 variceal band ligation VBL (16 months). Registrar 2 had performed 242 OGDs, one VBL (24 months). All patients provided written consent. Pharyngeal anaesthesia and/or intravenous sedation are not routinely used. Prospective collection of data included demographics, indications, endoscopic diagnosis and therapy.

Results Between the 2 UK StRs, 29 OGDs were performed in 4 days. These were both acute (14) and elective therapeutic (15) cases with a mean age of 51. 16 patients had VBL; 5 patients had sclerotherapy because of the unavailability of VBL; 1 patient had histoacryl glue injection of gastric varices; 1 had an oesophageal balloon dilatation. 6 patients did not require interventions. $55 \%(16 / 29)$ were related to periportal fibrosis; $14 \%$ (4/29) were related to hepatitis cirrhosis.

Table 1: UGI endoscopic therapeutics performed over 4 days Conclusion There is increasing cooperation between UK endoscopy and resource poor countries focusing on endoscopic training and support of their services. We have demonstrated that UK trainees can also benefit hugely from such interactions, both in terms of exposure to and skills acquisition from dealing with clinical problems relatively rare in UK training as well as experiencing the challenges of working in a resource poor healthcare setting. We would encourage these interactions as a mutually beneficial partnership. We plan to make the participation of our StRs a regular feature of our collaboration with SSG.

\section{PTH-138 EXPERIENCE FROM THE NATIONAL ERCP \& ENDOSCOPIC ULTRASOUND COURSE FOR NURSES}

Sister Sarah Jack*, Vanessa Chirnside, Kofi Oppong, John Leeds, Manu Nayar*. Pancreaticobiliary Endoscopy Unit, Freeman Hospital, Newcastle Upon Tyne, UK

\subsection{6/gutjnl-2018-BSGAbstracts.537}

Introduction ERCP and Endoscopic ultrasound (EUS) are complex endoscopic procedures requiring highly skilled nursing assistants. For these to be performed safely and efficiently, regular training and teaching of medical and nursing assistants is essential. In addition EUS has rapidly evolved into a therapeutic endoscopic procedure similar to ERCP. There are several training courses for the endoscopists but very few dedicated courses for nurses. Nurses feel less confident, out of touch and vulnerable in this rapidly evolving branch of endoscopy. The aim of the course is to:

- Educate nurses on the basic principles of the ERCP and EUS

- Share best practice in this field of endoscopy including recent advances in the field 